\title{
Research Paper \\ Effect of 8-Week Aerobic Exercise on Anthropometric Indices, Atherogenic Index of Plasma and Some Cardiovascular Risk Factors in Inactive Men
}

\author{
Marefat Siahkouhian ${ }^{1}$ (1), ${ }^{*}$ Bahman Ebrahimi-Torkmani ${ }^{1}$ (C)
}

1. Department of Exercise Physiology, Faculty of Physical Education \& Sport Sciences, University of Mohaghegh Ardabili, Ardabil, Iran.

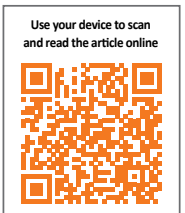

Citation Siahkouhian M, Ebrahimi-Torkmani B. Effect of 8-week Aerobic Exercise on Anthropometric Indices, Atherogenic Index of Plasma and Some Cardiovascular Risk Factors in Inactive Men. Scientific Journal of Rehabilitation Medicine. 2021; 10(3):446-457. https://doi.org/10.32598/sjrm.10.3.6

dol'https://doi.org/10.32598/sjrm.10.3.6

Received: 15 May 2020 Accepted: 22 July 2020 Available Online: $23 \mathrm{Jul} 2021$

Keywords:

Aerobic exercise, Lipids, Apolipoprotein, Cardiovascular diseases

\begin{abstract}
Background and Aims The sedentary lifestyle is related to the incidence of various diseases and metabolic disorders. Atherogenic Index of Plasma (AIP) can be considered as an appropriate criterion predicting coronary heart disease. This study aimed to investigate the Effect of 8-week aerobic training on anthropometric Indices, the Atherogenic Index of Plasma, and some cardiovascular risk factors in inactive men. Methods In the current quasi-experimental study, 40 inactive employees with a Mean \pm SD age of $36.17 \pm 8.97$ years and body mass index of $26.60 \pm 4.12 \mathrm{~kg} / \mathrm{m}^{2}$ after obtaining testimonial voluntarily performed aerobic exercise training for 8 weeks (each session 45 to 60 minutes) with 70 percent of maximal heart rate. Blood samples were taken before and after exercise to measure serum variables. Data were analyzed by paired t-test at the significant level of $P<0.05$.

Results Statistical analysis showed that after 8 weeks of aerobic exercise, Body fat percentage $(P=0.04)$, cholesterol $(P=0.001)$, $L D L / H D L$ ratio $(P=0.03), T G / H D L$ ratio $(P=0.01), T C / H D L$ ratio $(P=0.001)$, and apo- $B$ $(P=0.01)$ decreased significantly. Also, $\mathrm{VO}_{2} \max (P=0.011)$ and $\mathrm{HDL}(\mathrm{P}=0.010)$ increased dramatically after 8 weeks of participation in aerobic activity.

Conclusion According to the results, an increase in physical activity can probably reduce the risk of cardiovascular disease. Also, physical activity with lowering the risk factors of developing CVD events and significant prevention of its progression, the beneficial properties of physical activity are underscored, and promoting its effectiveness as support for healthier lifestyles in the community and particularly among inactive men is emphasized.
\end{abstract}

\section{Extended Abstract}

\section{Introduction}

ardiovascular Disease (CVD) is one of the main reasons for death worldwide and the leading reason for death in Iran. Although solid scientific evidence supports the posi- tive effects of regular exercise to prevent and manage CVD, sedentary lifestyles are prevalent worldwide.

A sedentary lifestyle is related to the incidence of various diseases and metabolic disorders. One-third of the world's population over the age of 15 does not get enough physical activity, which affects their health. A sedentary lifestyle is spreading worldwide for reasons such as lack of available spaces for exercise, increasing sedentary work behaviors

……

* Corresponding Author:

Bahman Ebrahimi-Torkmani, PhD student.

Address: Department of Exercise Physiology, Faculty of Physical Education \& Sport Sciences, University of Mohaghegh Ardabili, Ardabil, Iran.

E-Mail: ebrahimi.ba96@yahoo.com 
such as office work, and increasing use of devices such as cell phones and televisions. A sedentary lifestyle affects the human body through various mechanisms. Sedentary lifestyles reduce lipoprotein lipase activity, muscle glucose, protein transporter activities, impair lipid metabolism, and diminish carbohydrate metabolism. In addition, a sedentary lifestyle reduces cardiac output and systemic blood flow while activating the sympathetic nervous system, ultimately reducing insulin sensitivity and vascular function.

A risk factor that is (directly) associated with CVD is an Atherogenic Index of Plasma (AIP). The AIP can be considered as an appropriate criterion predicting coronary heart disease. AIP is also an indirect parameter indicating LowDensity Lipoprotein (LDL) particle size, and small-density LDL (sdLDL), a vital risk factor for atherosclerosis. It is a more sensitive factor that predicts cardiovascular events.

On the other hand, regular exercise is one of the factors determining weight reduction and fat loss, and at the same time, it is associated with essential health benefits. This study aimed to investigate the Effect of 8-week aerobic training on anthropometric Indices, the Atherogenic Index of Plasma, and some cardiovascular risk factors in inactive men.

\section{Methods}

In the current quasi-experimental study, Forty inactive employees with a Mean \pm SD age of $36.17 \pm 8$ years and body mass index of $26.60 \pm 4 \mathrm{~kg} / \mathrm{m}^{2}$ after obtaining testimonial voluntarily performed aerobic exercise training for 8 weeks (each session 45 to 60 minutes) with 70 percent of maximal heart rate. None of the subjects participated in regular exercise activities in the 3 years leading up to the study. All subjects completed a written consent form before starting the exercise program. Fasting blood samples to measure serum levels of cholesterol, High-Density Lipoprotein (HDL), Low-Density Lipoprotein (LDL), triglyceride, Apolipoprotein A (apoA), Apolipoprotein B (apoB) were obtained from the subjects by a laboratory expert at 7:30-8 AM 24 hours before and 48 hours after the training protocol. In addition, all subjects' $\mathrm{VO}_{2} \max$, Body Fat Percentage, and Waist-to-Hip Ratio (WHR) index were measured. Data were analyzed by paired t-test at the significant level of $\mathrm{P}<0.05$.

\section{Results}

Statistical analysis showed that after 8 weeks of aerobic exercise, Body fat percentage $(\mathrm{P}=0.04)$, cholesterol $(\mathrm{P}=0.001), \mathrm{LDL} / \mathrm{HDL}$ ratio $(\mathrm{P}=0.03), \mathrm{TG} / \mathrm{HDL}$ ratio $(\mathrm{P}=0.01), \mathrm{TC} / \mathrm{HDL}$ ratio $(\mathrm{P}=0.001)$, and apo- $\mathrm{B}(\mathrm{P}=0.01)$ and subjects resting heart rate $(\mathrm{P}=0.001)$ decreased sig- nificantly. Also, $\mathrm{VO}_{2} \max (\mathrm{P}=0.010)$ and $\mathrm{HDL}(\mathrm{P}=0.010)$ increased dramatically after 8 weeks of participation in aerobic training. But no significant change was observed in apo-A, triglyceride, low-density lipoprotein, and body mass index $(\mathrm{P}<0.05)$.

This study aimed to determine the effects of 8-week aerobic exercise on AIP, apoA, apoB, and lipid profile in inactive men. The study results showed that regular participation in aerobic exercise increases the process of reverse cholesterol transfer and improves the rate of blood lipid profiles in inactive men.

The TG/HDL-C logarithm, as an atherogenic indicator, represents the equilibrium between the actual plasma TG concentration and HDL-C, which determines the cholesterol transport pathway within the arteries. As the TG/HDL-C ratio increases, HDL particles tend to become smaller in size. Therefore, a high ratio indicates the possibility of stopping HDL-C and weakening the reverse cholesterol transfer. On the other hand, decreasing the ratio of triglycerides to high-density lipoprotein may be more likely due to lower cholesterol due to the improvement of some key factors in the reverse cholesterol transport process, such as increased ABCA1 transporters increased Lecithin Cholesterol Acyl Transferase (LCAT). The Effect of exercise on LDL and HDL-C levels varies depending on the characteristics of the exercise program such as intensity, duration and repetition, and the increase in HDL-C levels generally occurs in high-intensity exercise programs, so one of the reasons for the lack of significant increase HDL-C as well as lack of significant reduction in LDL can be the average intensity of the exercise program.

\section{Discussion and Conclusion}

Of the Effect of exercise on the lipid profile, several points can be identified: A review of research in this field shows that the duration of aerobic exercise is an essential factor so that the effectiveness of protocols that have used long-term training (more than 12 weeks) is more than short-term exercise programs. Second, it has been reported in many studies that weight-free physical activity can also have beneficial effects on the lipid profile, and weight loss does not require changes in plasma lipoproteins. Thirdly, physical activity will not affect the lipid profile of people who have regular levels of these indicators. According to the present study results, increasing the levels of physical activity effectively reduces the risk of cardiovascular disease and physical activity as a supportive measure to enjoy a healthier lifestyle in society, significantly Benefited among employees and inactive people. 


\section{Ethical Considerations}

Compliance with ethical guidelines

\section{All ethical principles are considered in this article}

Funding

This research did not receive any grant from funding agencies in the public, commercial, or non-profit sectors.

Authors' contributions

The authors contributed equally in preparing this article.

Conflict of interest

The authors declared no conflict of interest. 
مقاله تاثير هشت هفته تمرين هوازى بر شاخص هالى تنسنجى، آتروزنيك يالاسما و برخى عوامل

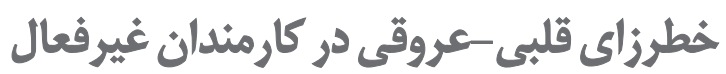

معرفت سياكوهيان' هـ، "بهمن ابراهيمى تركمانى '

1. كروه فيزيولورثى ورزشى، دانشكده تربيتبنىى و علوم ورزشى، دائشعاه محقق اردبيلى، اردبيل، ايران.

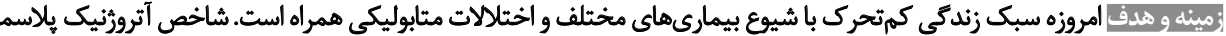

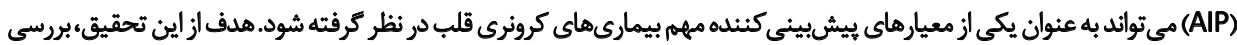

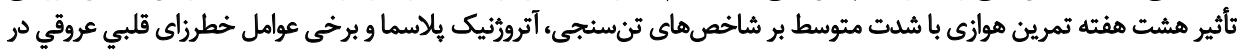

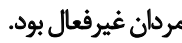

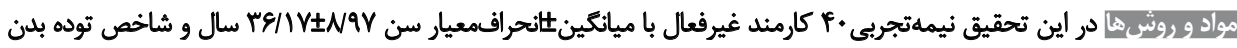

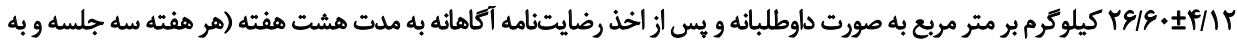

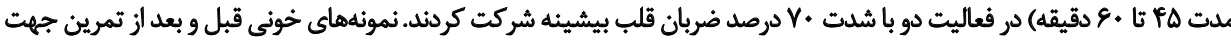

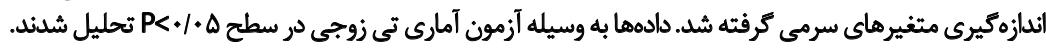

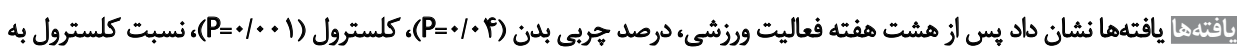

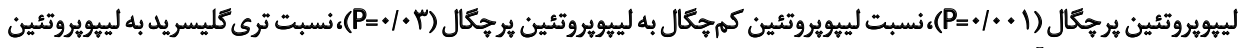

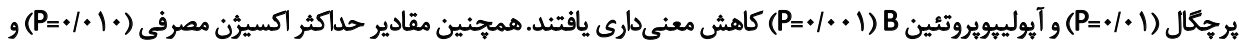

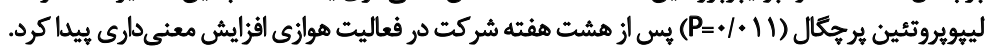

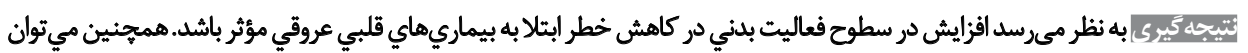

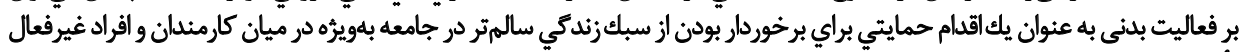
تأكيد كرن.

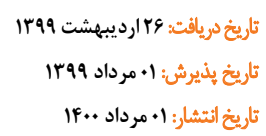

كليدوارئهائه

تمرين هوازي، يروفايل جيربى، آيولييويروثئين، بيمارى هاى قلبى عروقى آلى

فعاليت بدنى مناسبى ندارند احتمال ابتلا به بيمارىهاى قلبي

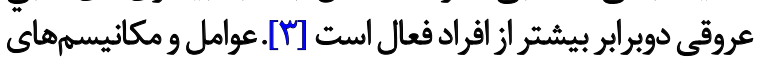

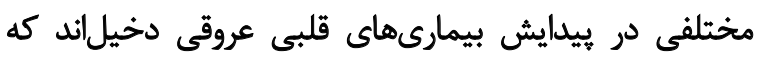

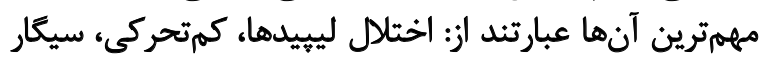

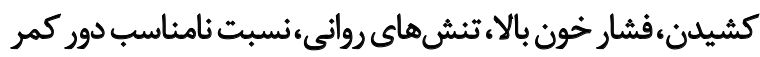

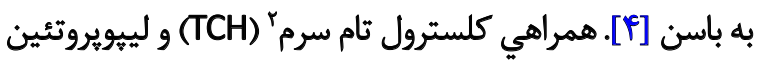

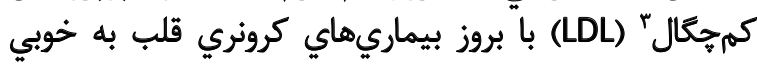

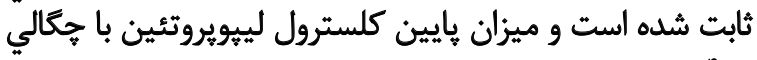

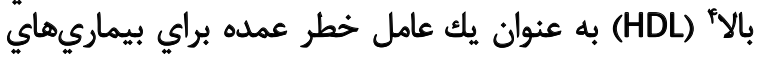

2. Total Cholesterol (TCH)

3. Low Density Lipoprotein (LDL)

4. High Density Lipoprotein (HDL)

مelat

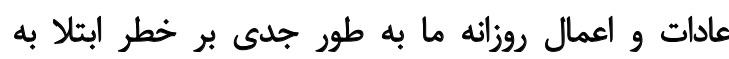

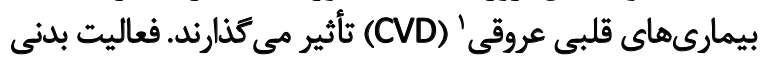

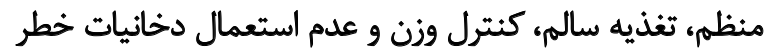

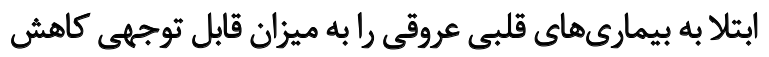

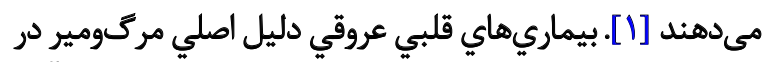

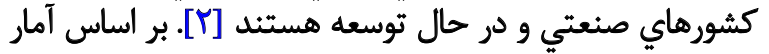

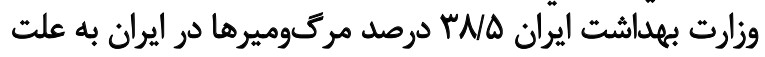

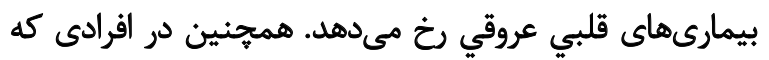

1. Cardiovascular Disease (CVD)

$$
\begin{aligned}
& \text { " نويسنده مسئول: } \\
& \text { بهمن ابراهيمى تركمانى }
\end{aligned}
$$

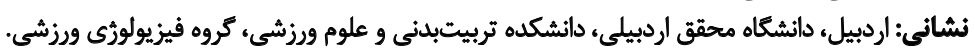
رايانامه: ebrahimi.ba96@yahoo.com 
انجمن قلب آمريكا' ' (AHA) فعاليت بدنى رابه عنوان يكى از هفت

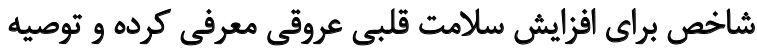

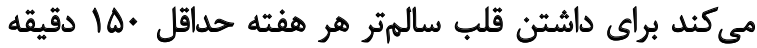

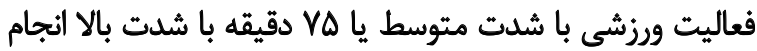

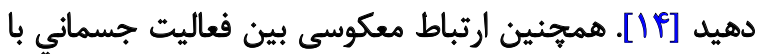

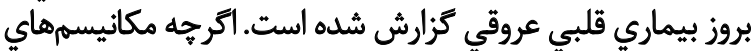

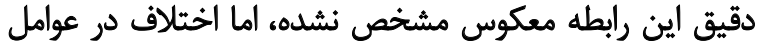

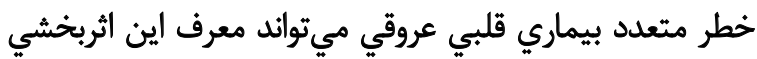

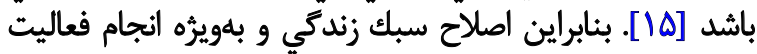

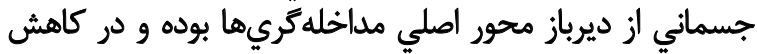

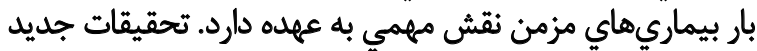

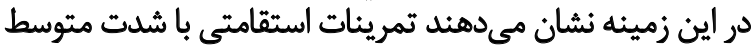

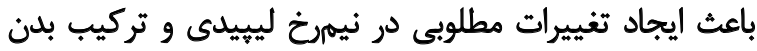

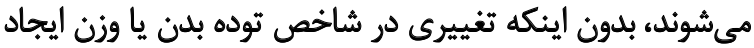

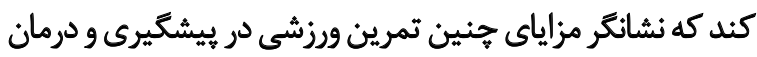

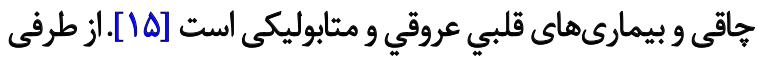

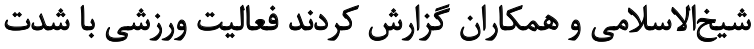

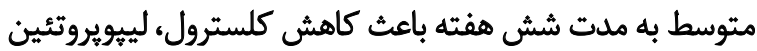

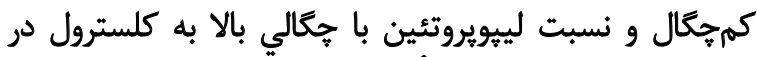

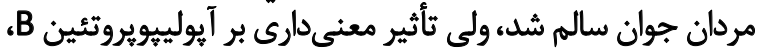

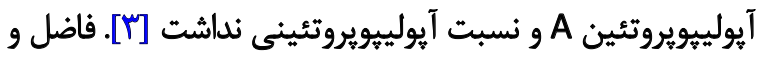

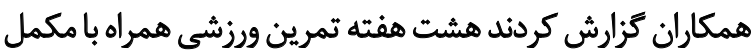

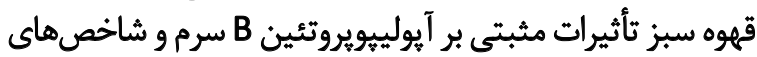

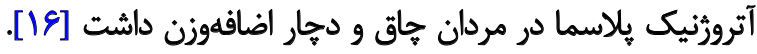

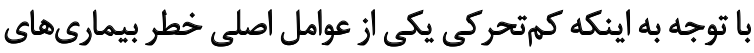

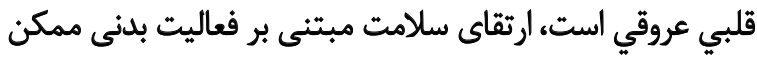

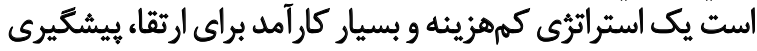

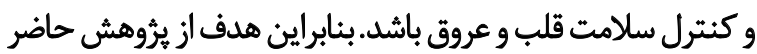

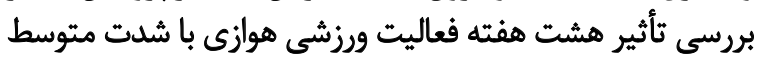

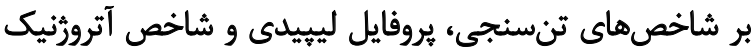

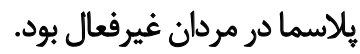

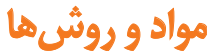

در اين تحقيق نيمهتجربى ·f نقر از كاركنان ادارى مرد

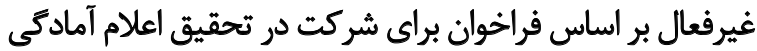

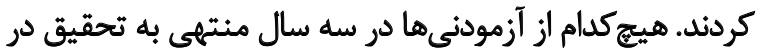

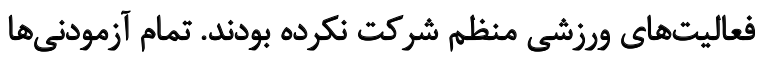

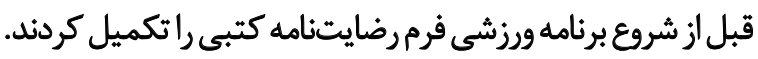

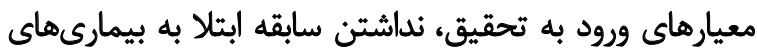

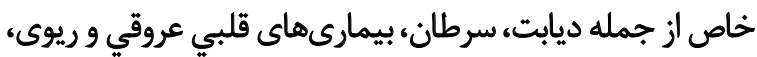

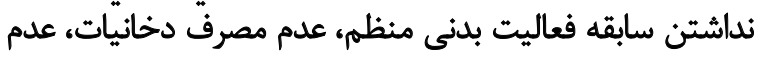

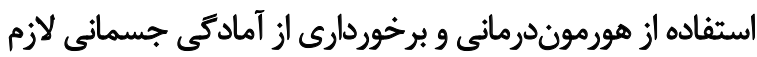

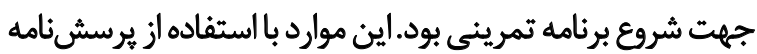

10. American Heart Association (AHA)
كرونري قلب در نظر كرفته ميشود [ه]. همجنين شواهدي

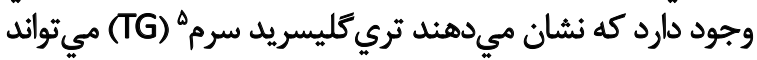

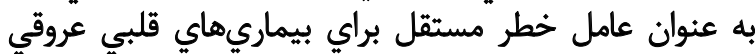

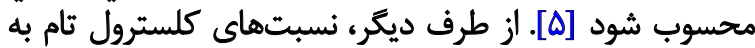

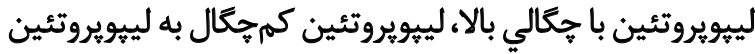

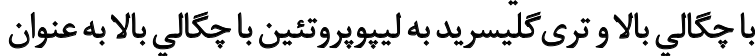

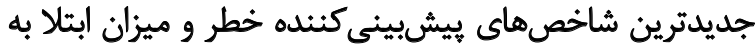

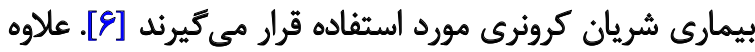

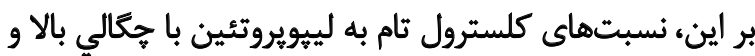

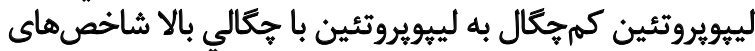

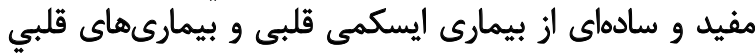

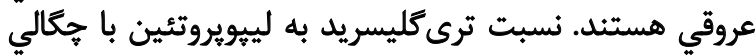

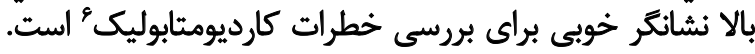

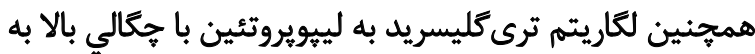

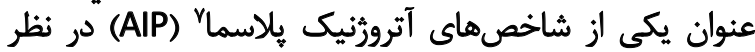

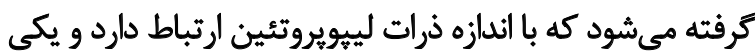

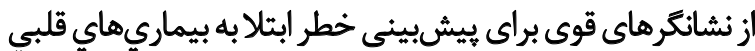

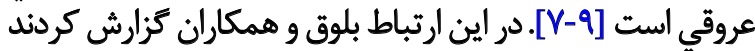

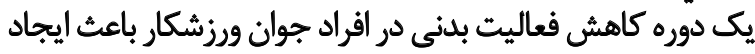

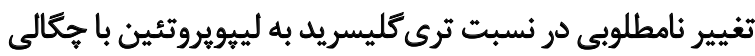

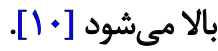

از طرف ديكر در دهلهاي اخير به منظور شناخت و بررسي

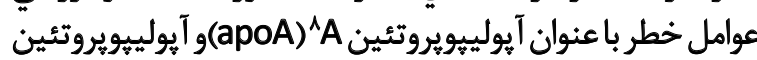

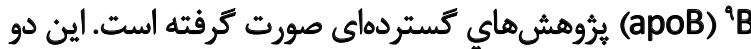

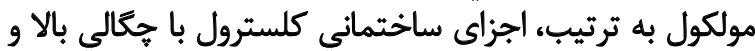

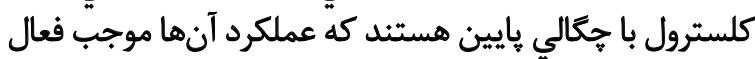

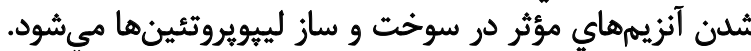

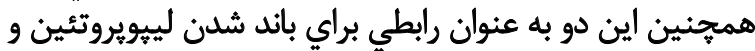

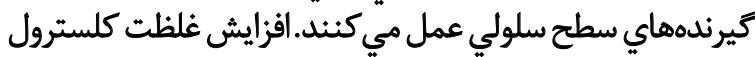

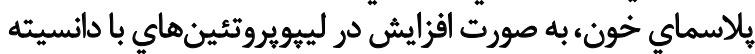

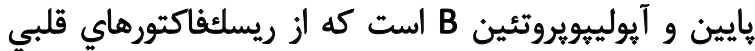

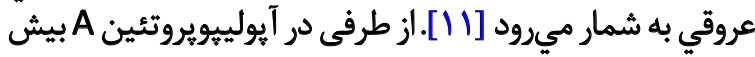

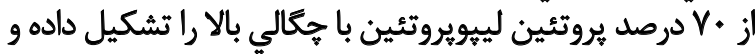

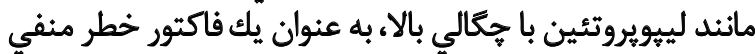

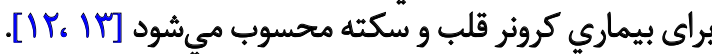

اين توافق كلي وجود دارد كه فعاليت جسماني با كاهش خطر بران

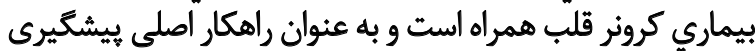

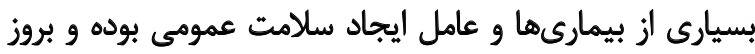

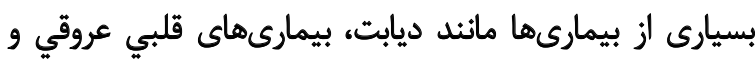

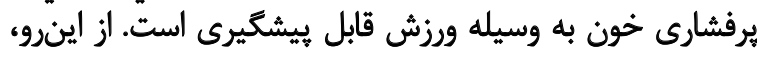

5. Triglyceride (TG)

6. Cardiometabolic

7. Atherogenic Index of Plasma (AIP)

8. Apolipoprotein A (apoA)

9. Apolipoprotein B (apoB) 
و كلسترولاكسيداز و براي اندازهيرى ترى كليسريد از آنزيمهاى

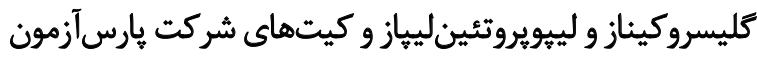

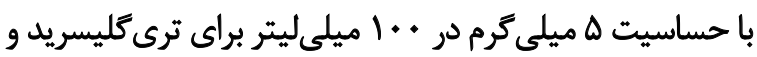

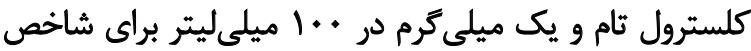

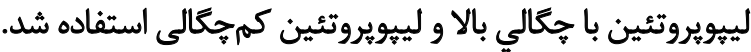

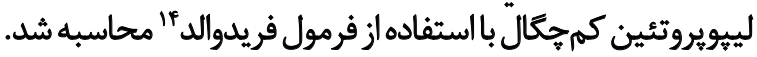

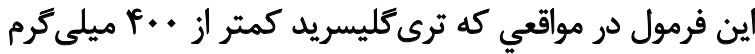

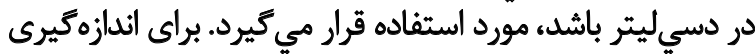

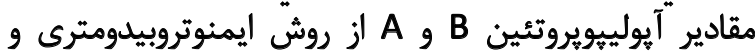

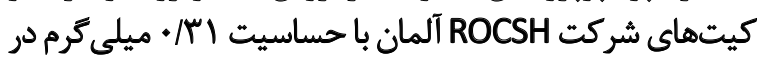

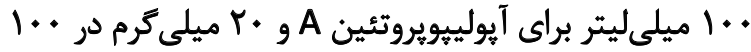
ميلى ليتر براي آيولييويروتئين B استفاده شد.

$$
\text { تجزئه و تحليل آمارى }
$$

كليه اطلاعات حاصل با استفاده از نرمافزار آمارى SPSS

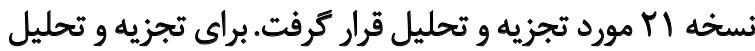

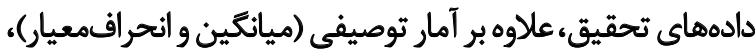

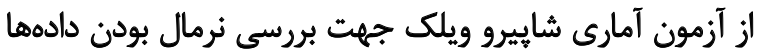

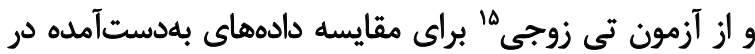

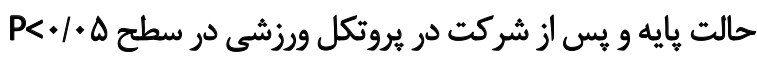

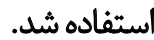

\section{يافتهانا}

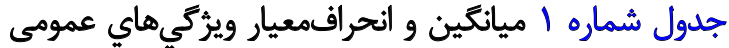

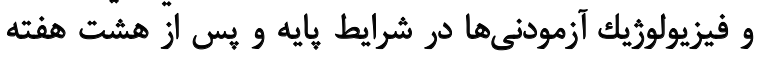
فعاليت ورزشى هوازى رانشان مي دهدئ

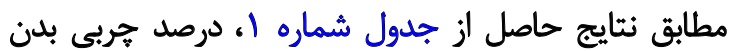

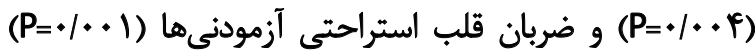

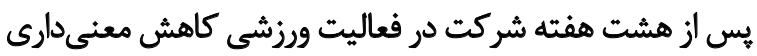

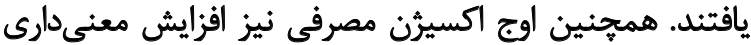

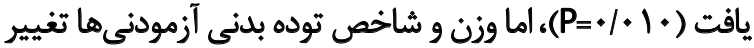

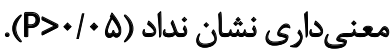

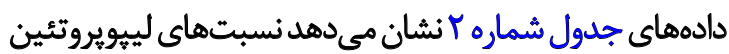

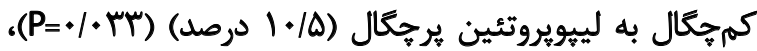

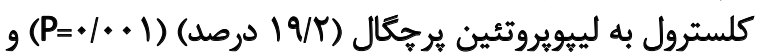

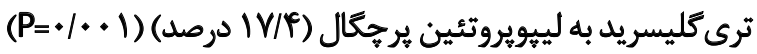

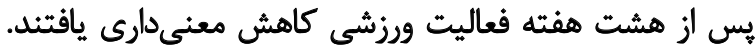

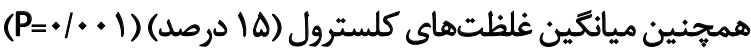

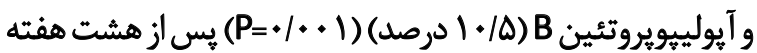

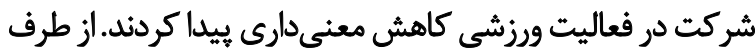

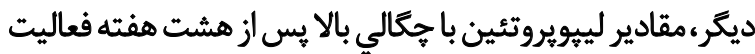

سابقه يزشكى كه توسط خود آزمودنىها تكميل شده بود مورد

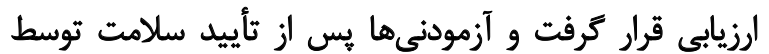

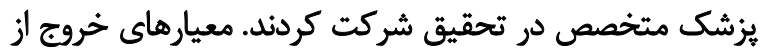

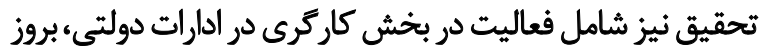

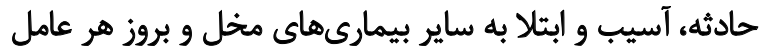

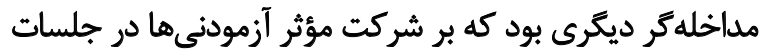

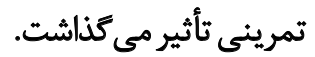

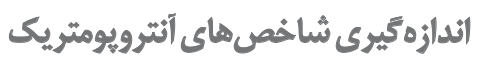

اندازهكيرى قد (سانتيمتر) و وزن (كيلوكرم) با استفاده از وزئ

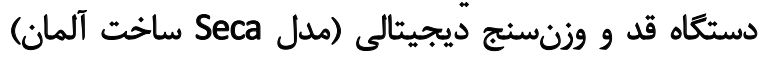

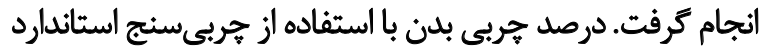

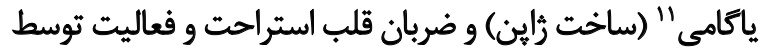

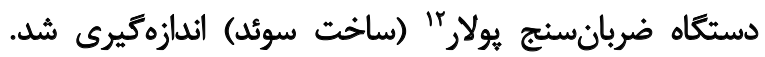

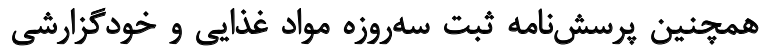
جهت ارزيابى بهتر شرايط و وضعيت غذايى آزمودنى ثنامها مورد

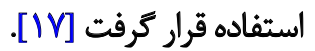

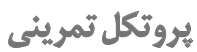

آزمودنى ها برنامه تمرينى را به مدت هشت هفته (سه جلسه

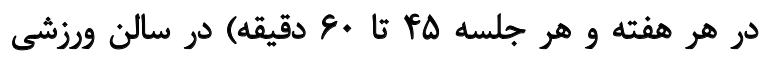

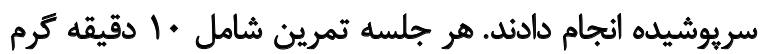

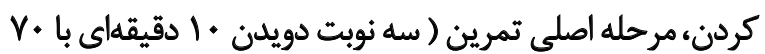

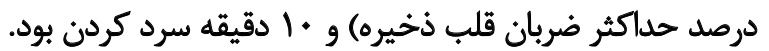

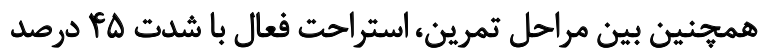
حداكثر ضربان قلب ذخيره صورت كرفت تمرين استراحت

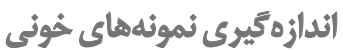

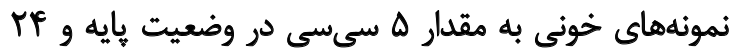

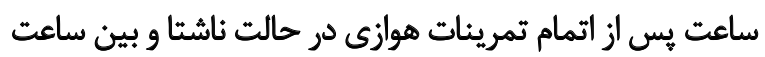

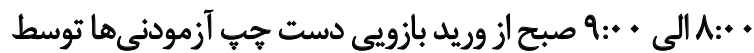

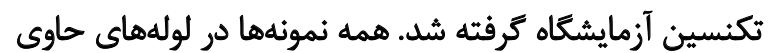

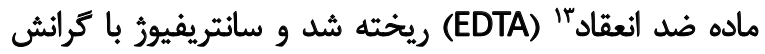

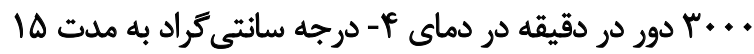

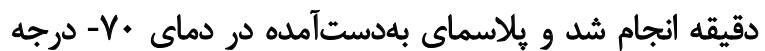
سانتى كراد قرار كرفت.

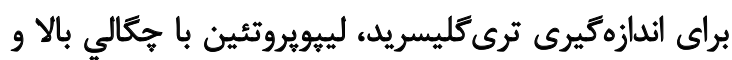

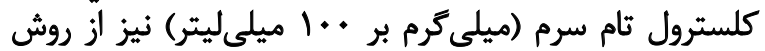

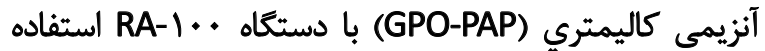
شد. براى اندازهيرى كلسترول تام ازئزي آنزيمهاى كلسترولاستراز

13. Ethylenediaminetetra-acetic Acid (EDTA) 
جدول ا. ميانكين و انحرافمعيار ويرُكى هاى عمومى و فيزيولوزيكى آزمودنىها در حالت بايه و پِّ از هشت هفته فعاليت ورزشى

\begin{tabular}{|c|c|c|c|}
\hline \multirow[b]{2}{*}{ سطح معنى دارى } & \multicolumn{2}{|c|}{ مياتكين } & \multirow{2}{*}{ 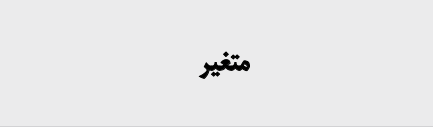 } \\
\hline & مرحله دوم & حالت بايه & \\
\hline & - & rg/IY $\pm N q Y$ & سن (سال) \\
\hline & - & $I V \cdot / r \cdot \pm \Delta / / r$ & قد (سائتى متر) \\
\hline .10 & $Y \& / q \varepsilon \pm 1 / q T$ & $W / 1 F \pm T / \cdot \Delta$ & وزن (كيلوكرم) \\
\hline$\cdot / \Delta \cdot r$ & 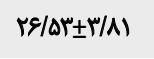 & $r \& / 8 \cdot \pm F / M$ & شاخص توده بدن (كيلوكرم بر هتر هربع) \\
\hline $.10 .+\circ$ & If/RT+/Ar & $\mid E / M+1 / M$ & هربى (درصل) \\
\hline $.1 \cdot 1 *^{\circ}$ & rNTTEI/IT & $r g / \Delta f \pm 1 / \cdot r$ & اوج اكسيثن مصرفى (ميلى ليتر / كيلوكرم بر دقيقه) \\
\hline $.1 . .11^{*}$ & $Y V / I T \pm / / M$ & $V V \Delta \Delta \cdot \pm V / A r$ & ضربان ثلب استراحت (ضريه در دقيقه) \\
\hline
\end{tabular}

كلسترول به لييويروتئين يرجحال، تري كليسيريد به ليويويروتئين

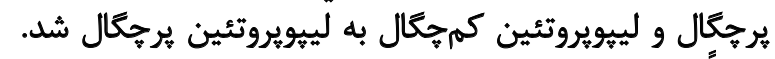

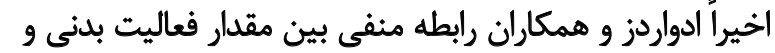

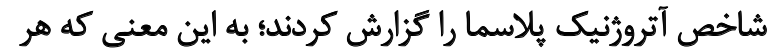

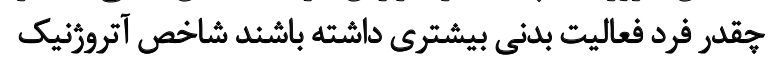



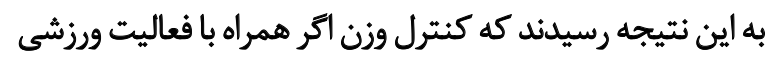

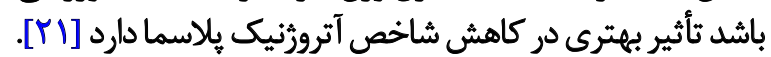

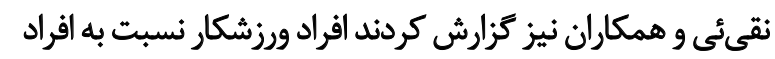

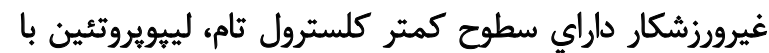

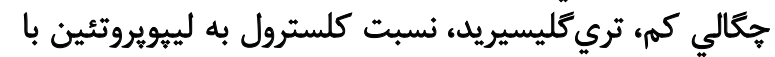

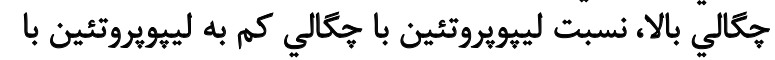

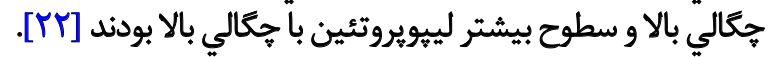

فعاليت ورزشى هوازى از طريق افزايش آنزيم ليبويروتئينيهاز

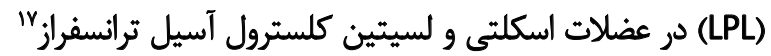

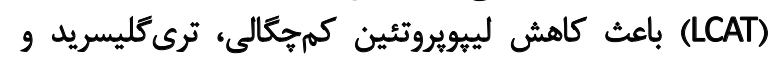

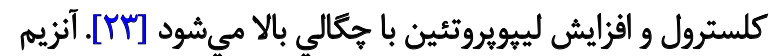

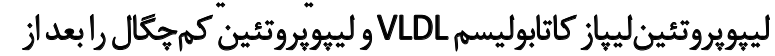

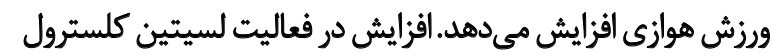

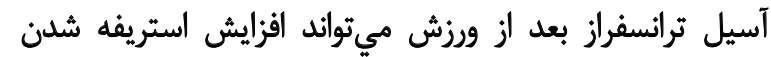

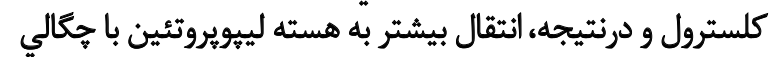

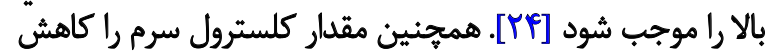

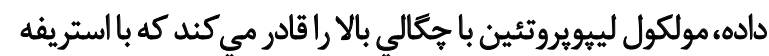

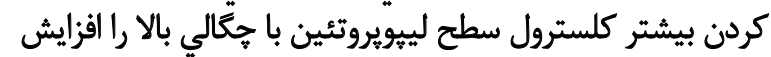

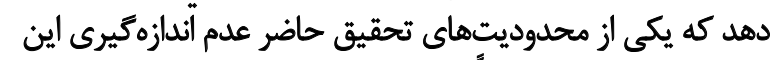

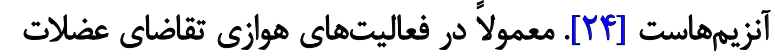

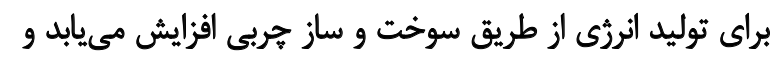

16. Lipoprotein Lipase (LPL)

17. Lecithin-Cholesterol Acyltransferase (LCAT)
ورزشى افزايش معنى دارى يافت (ع درصد) (11 (1)

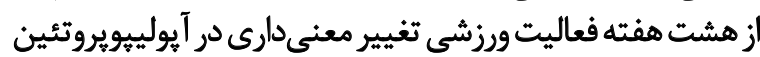

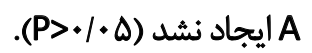

\section{ث}

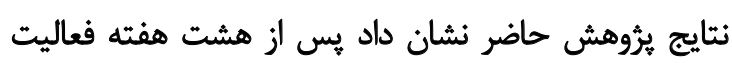

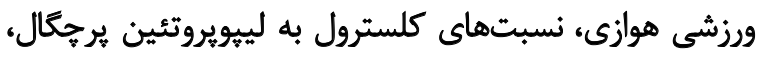

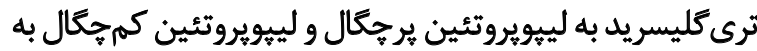

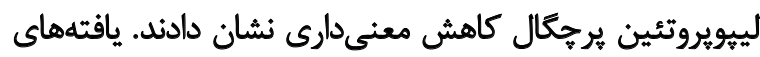

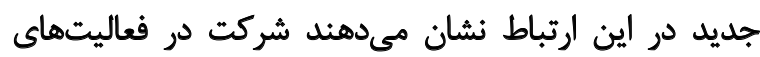

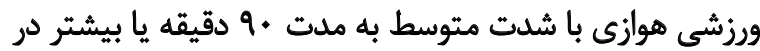

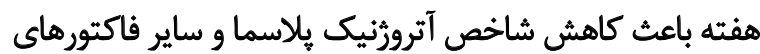

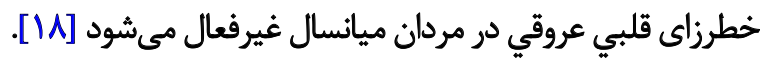

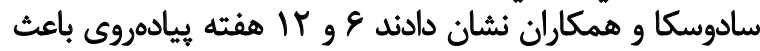

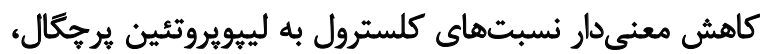

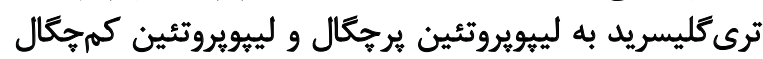

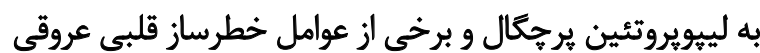

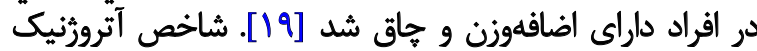

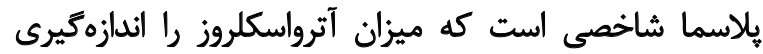

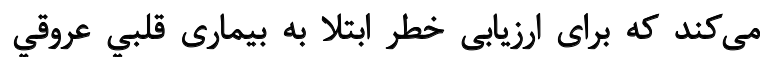

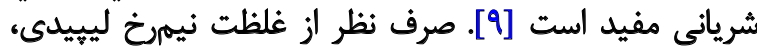

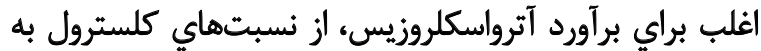

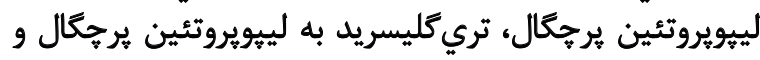

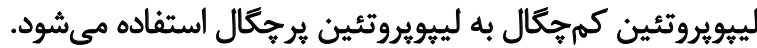

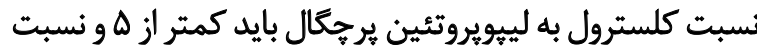

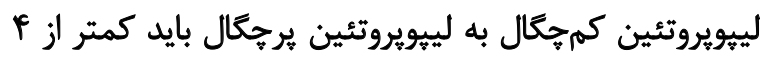

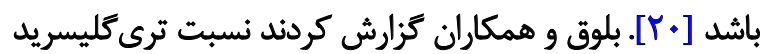

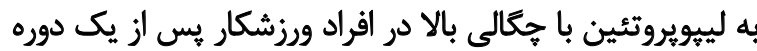

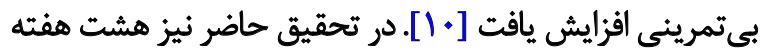

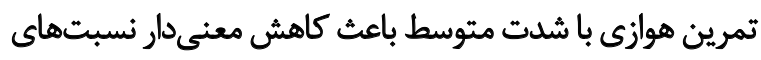


جدول ب. مقايسه مقادير متغيرهاى وابسته در مراحل يُيش آزمون و يُ آزمون با استفاده از آزمون تى زوجى

\begin{tabular}{|c|c|c|c|}
\hline \multirow{2}{*}{ سطح معنى دارى } & \multicolumn{2}{|c|}{ مياتكين+|نحراثنمعيار } & \multirow{2}{*}{ مثغير } \\
\hline & مرحله دوم & حالت هايه & \\
\hline . & $\| g / V^{\prime} \pm M V / g t$ & $\mid W Q I \pm T / \& S$ & لييويروتئن كمجكالى (ميلى كرم بر دسىليير) \\
\hline.$/ 1 \cdot Y$ & $\mid \varnothing V / \% \Delta \pm 1 . / \Delta V$ & $19 T / 4 A \pm Y+/ 48$ & ترى كليسيريد (ميلى كرم بر دسىليتر) \\
\hline $.1 \cdot 11^{*}$ & $r \Delta / P q \pm / T F$ & $M / R I N A R$ & لييويروتئين يرجكال (ميلى كرم ير دسىليثر) \\
\hline $.1 . .1^{\circ}$ & $|\Delta| / T \Delta \pm \mu \varphi / T q$ & IVNYAETM/IT & كلسترول (ميلى كرم بر دسى ليتر) \\
\hline $.1 \cdot .1^{*}$ & $f / f \Delta \pm \mid / F)$ & $\Delta / \Delta I \pm T / T)$ & نسبت كلسترول به لييويروتئين يرجكال \\
\hline $1 . .1 *$ & $\Delta / 11 \pm F / T \Lambda$ & 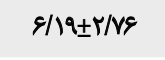 & نسبت ترى كليسيريد به لييويروتئن برجكال \\
\hline $.1 \cdot m *$ & $r / R Y \pm 1 / r$. & $r / M \pm V / \Delta V$ & نسبت لييويروتئين كمجال به لييويروتئين يرجكال \\
\hline$\cdot / \mathrm{Ar}$ & $\mid \Delta E / V A \pm T \Delta / \mathrm{TA}$ & $10 V / \pi f \pm F / / A V$ & آيولييويروتئين A \\
\hline $.1 .01^{*}$ & $\Delta Q / \Delta q \pm 1 \Delta / \Delta T$ & $1.0 / 10 \pm r V / \Delta A$ & آيولييويروتئين B \\
\hline
\end{tabular}

تغييرات معنى دار در ميزان كلسترول، تري كليسريد، لييويروتئين

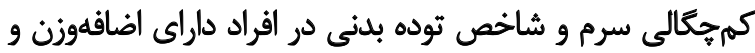

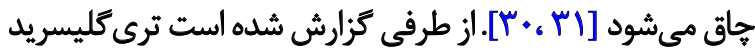

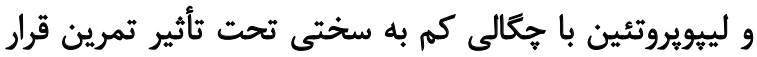

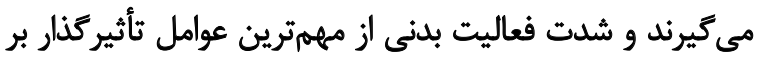

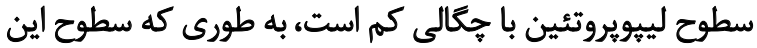

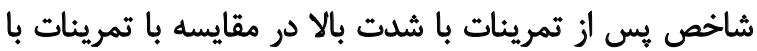

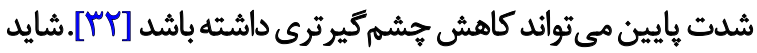

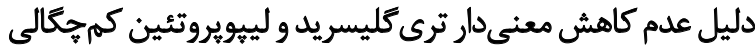

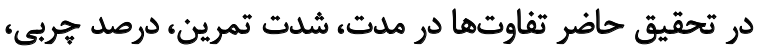

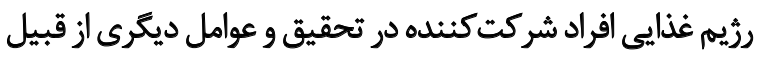

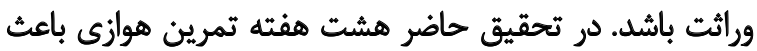

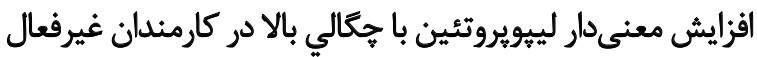

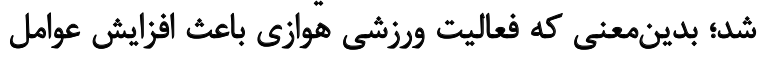

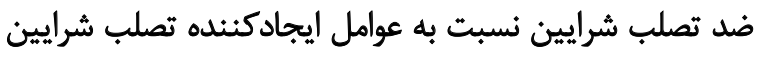

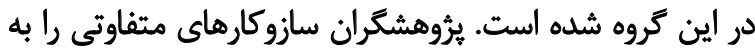

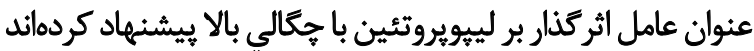

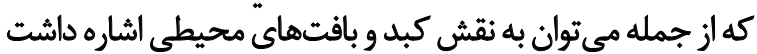

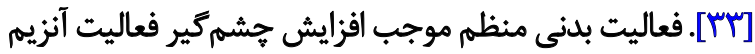

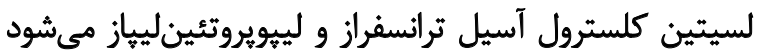

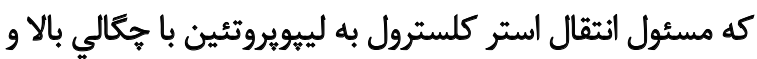

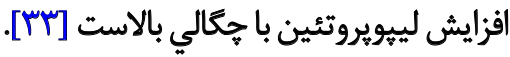
از طرفى در تحقيق حاضر هشت هفته تمرين هوازى باعث

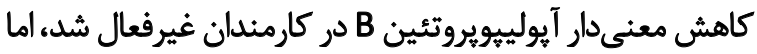

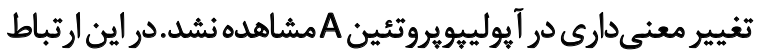

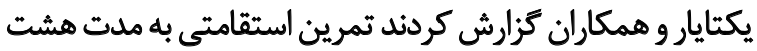
هفته تأثير معنى دارى بر سطوح آيولييويروتئين A، آيولييويروتئين
بنابراين اكسيداسيون جربى بيشتر مىشود. از طرفى در اينكونه

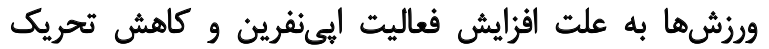

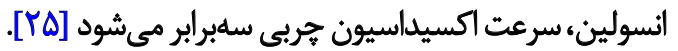
در تحقيق حاضر نيز يس از هشت هفته فعاليت هوازى درصد

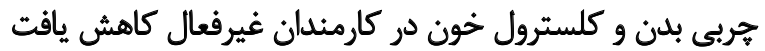

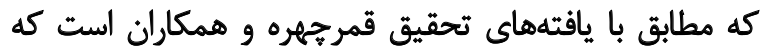
كزارش كردند هشت هفته تمرين هوازى باعث كاهش كائ كلسترول

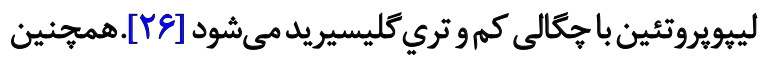

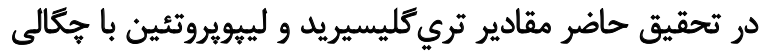

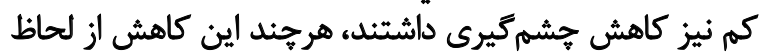

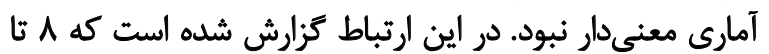

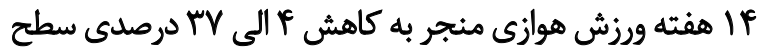

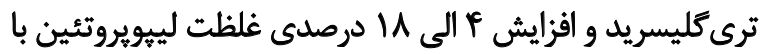

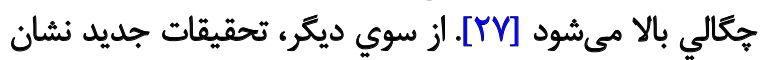

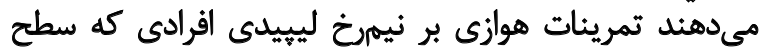

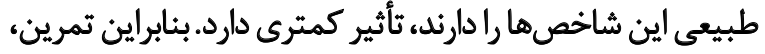

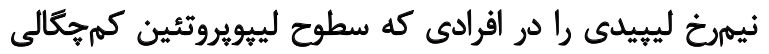

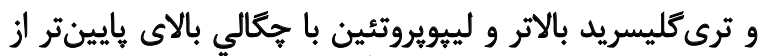

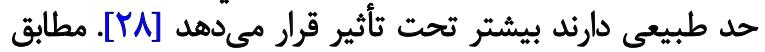

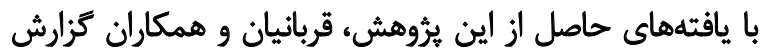

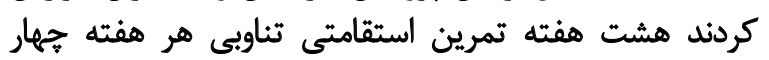

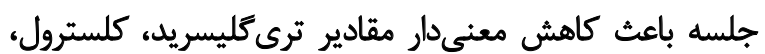

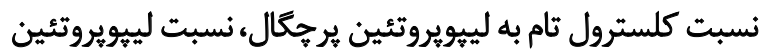

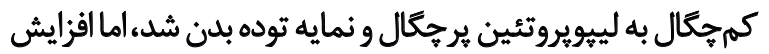

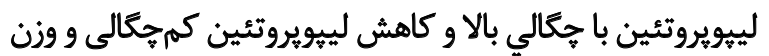

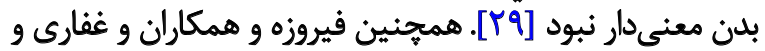

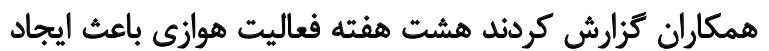




\section{تعارض مثافع}

بنابر اظهار نويسندكان اين مقاله تعارض منافع ندارد.

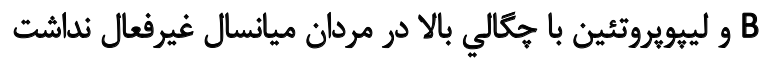

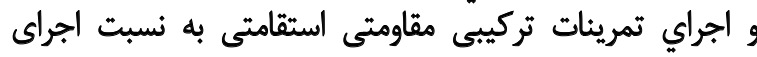

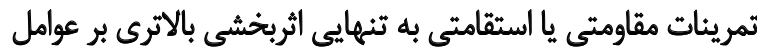

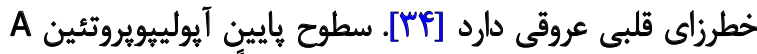

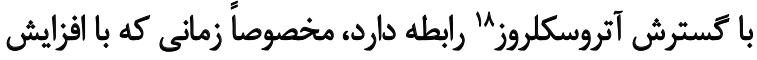

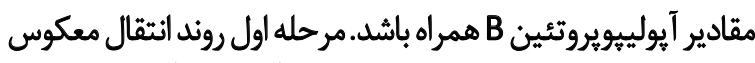

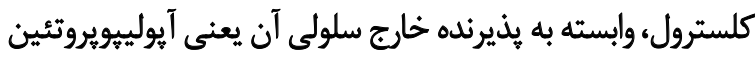

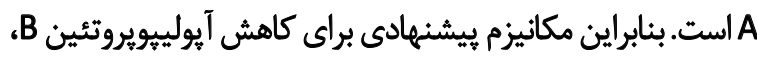

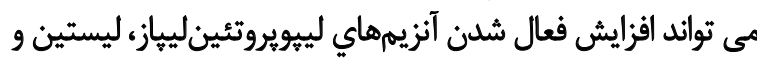

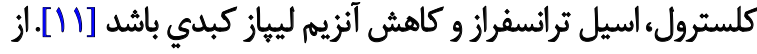

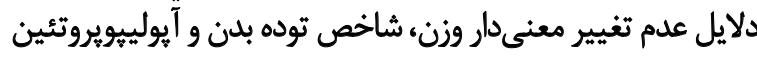

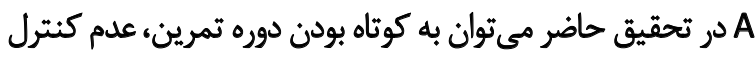

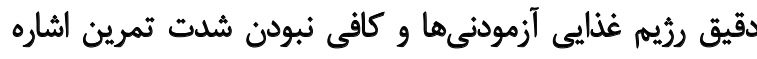

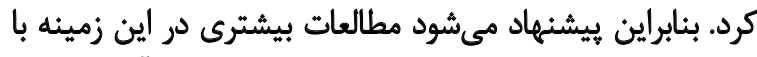

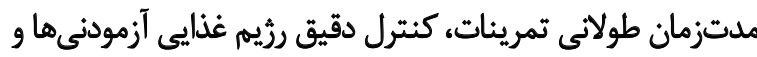

$$
\text { تمرين با شدت بالاصورت كيرد. }
$$

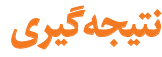

در حالت كلى يافتههاى برّوهش حاضر نشان داد هشت هفته

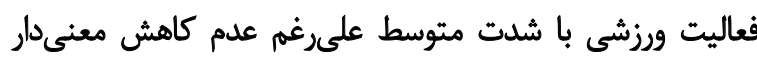

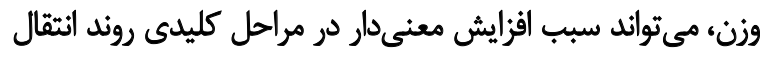

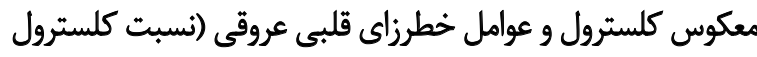

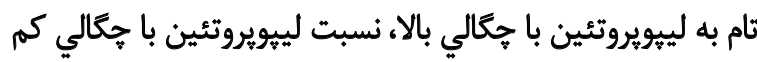

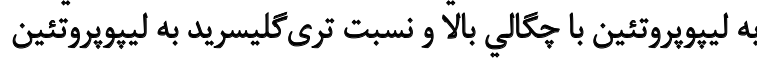

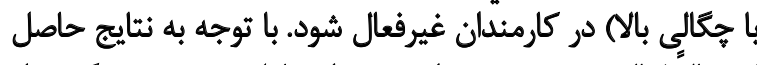

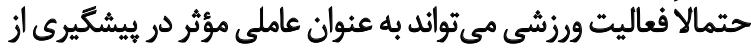

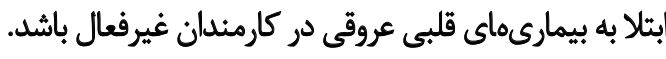

مالاحظات أخالاقي

$$
\text { يميروى أز اصول اخلاق يثووهش }
$$

در اجراي يثوهش، ملاحظات اخلاقى مطابق بادستورالعمل كميته اخلاق دانشعاه محقق اردبيلى در نظر ترفته شده است ماتئ

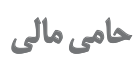

اين مقاله هيجّونه كمك مالى از سازمان تأمينكنيده مالى در

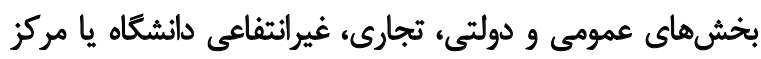
تحقيقات دريافت نكرده است

$$
\text { مشاركت نويسيندكان }
$$

تمام نويسندكان در آمادهازى اين مقاله مشاركت يكسان

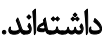

18. Atherosclerosis 


\section{References}

[1] Rippe JM. Lifestyle strategies for risk factor reduction, prevention, and treatment of cardiovascular disease. American Journal of Lifestyle Medicine. 2019; 13(2):204-12. [DOI:10.1177/1559827618812395] [PMID] [PMCID]

[2] Fathei M, Khairabadi S, Ramezani F, Hejazi K. [The effects of eight weeks aerobic training, green tea supplementation and compound of them on serum liver enzymes and apolipoproteins in inactive overweight women (Persian)]. Medical Journal of Mashhad University of Medical Sciences. 2016; 59(2):114-23. http://eprints.mums.ac.ir/3888/

[3] Sheykholeslami Vatani D, Ahmadi S, Mojtahedi H, Marandi M, Ahmadi Deharshid K, Faraji H, et al. [Effect of moderate and high intensity resistant exercises on cardiovascular risk factors in non-athlete university students (Persian)]. Kowsar Medical Journal. 2011; 16(2):115-21. https://www.sid.ir/en/journal/ ViewPaper.aspx?id=199787

[4] Nystoriak MA, Bhatnagar A. Cardiovascular effects and benefits of exercise. Frontiers in Cardiovascular Medicine. 2018; 5:135. [DOI:10.3389/fcvm.2018.00135]

[5] Khalili D, Hadaegh F, Tohidi M, Ghasemi A, Sheikholeslami F, Azizi F. Triglyceride/HDL-cholesterol ratio (TG/HDL-C) beside the total cholesterol is a predictor for coronary heart disease in an Iranian men population. Iranian Journal of Epidemiology. 2009; 4(3):77-86. https://irje.tums.ac.ir/browse.php?a_ id=136\&sid=1\&slc_lang=en

[6] Chuang TL, Lin JW, Wang YF. Bone mineral density as a predictor of atherogenic indexes of cardiovascular disease, especially in nonobese adults. Disease Markers. 2019; 2019:1045098. [DOI:10.1159/000345461] [PMID] [PMCID]

[7] Wu TT, Gao Y, Zheng YY, Ma YT, Xie X. Atherogenic Index of Plasma (AIP): A novel predictive indicator for the coronary artery disease in postmenopausal women. Lipids in Health and Disease. 2018; 17(1):197. [DOI:10.1186/s12944-018-0828-z] [PMID] [PMCID]

[8] Miralles CSW, Wollinger LM, Marin D, Genro JP, Contini V, Dal Bosco SM. Waist-to-height ratio (WHtR) and triglyceride to HDL-C ratio (TG/HDL-c) as predictors of cardiometabolic risk. Nutrición Hospitalaria. 2015; 31(5):2115-21. [doi:10.3305/ nh.2015.31.5.7773]

[9] Guo Q, Zhou S, Feng X, Yang J, Qiao J, Zhao Y, et al. The sensibility of the new blood lipid indicator Atherogenic Index of Plasma (AIP) in menopausal women with coronary artery disease. Lipids in Health and Disease. 2020; 19(1):27. [DOI:10.1186/ s12944-020-01208-8] [PMID] [PMCID]

[10] Blough J, Loprinzi PD. Randomized controlled trial investigating the experimental effects of reduced habitual physical activity on cardiometabolic profile. Physiology \& Behavior. 2018; 194:48-55. [DOI:10.1016/j.physbeh.2018.04.036] [PMID]

[11] Pourvaghar MJ, Shahsavar A, Bahram ME. [The effect of a single bout of severe aerobic exercise on apolipoproteins A, B and some serum lipid profiles (Persian)]. Feyz. 2015; 18(6):585-91. http://feyz.kaums.ac.ir/browse.php?a_id=2463\&sid=1\&slc lang=en\&ftxt=1
[12] Suter E, Marti B, Tschopp A, Wanner H-U, Wenk C, Gutzwiller F. Effects of self-monitored jogging on physical fitness, blood pressure and serum lipids: A controlled study in sedentary middle-aged men. International Journal of Sports Medicine. 1990; 11(06):425-32. [DOI:10.1055/s-2007-1024832] [PMID]

[13] Guyton AC. Textbook of medical physiology. $2^{\text {th }}$ ed. Philadelphia: Saunders; 1961. https://www.worldcat.org/title/textbookof-medical-physiology/oclc/1399546?referer=di\&ht=edition

[14] Lloyd-Jones DM, Hong Y, Labarthe D, Mozaffarian D, Appel LJ, Van Horn L, et al. Defining and setting national goals for cardiovascular health promotion and disease reduction: The American Heart Association's strategic Impact Goal through 2020 and beyond. Circulation. 2010; 121(4):586-613.[DOI:10.1161/CIRCULATIONAHA.109.192703] [PMID]

[15] Tartibian B, Kushkestani M, Ebrahimpour Nosrani S. The effect of 12-week endurance training on lipid profiles and fat percentage of overweight girls. New Approaches in Sport Sciences. 2019; 1(1):189-200. [DOI:10.22054/nass.2019.10134]

[16] Fazel F, Naghibzadeh A, Mohammad Ramezanpour R, Bagheri $R$, Hamidi A, Rashidlamir A. Effect of aerobic exercise with and without green coffee supplementation on serum apolipoprotein $B$ and atherogenic indices of overweight men. Medical Laboratory Journal. 2019; 13(3):20-4. [DOI:10.29252/mlj.13.3.20]

[17] Esfahani FH, Asghari G, Mirmiran P, Azizi F. Reproducibility and relative validity of food group intake in a food frequency questionnaire developed for the Tehran Lipid and Glucose Study. Journal of Epidemiology. 2010; 20(2):150-8. [DOI:10.2188/jea. JE20090083] [PMID] [PMCID]

[18] Shen S, Qi H, He X, Lu Y, Yang C, Li F, et al. Aerobic exercise for a duration of $90 \mathrm{~min}$ or longer per week may reduce the atherogenic index of plasma. Scientific Reports. 2018; 8(1):1730. [DOI:10.1038/s41598-018-20201-x] [PMID] [PMCID]

[19] Sadowska-Krępa E, Gdańska A, Rozpara M, Pilch W, Přidalová $\mathrm{M}$, Bańkowski S. Effect of 12-week interventions involving nordic walking exercise and a modified diet on the anthropometric parameters and blood lipid profiles in overweight and obese ex-coal miners. Obesity Facts. 2020; 13(2):201-12. [DOI:10.1159/000506403] [PMID] [PMCID]

[20] Edwards MK, Blaha MJ, Loprinzi PD. Influence of sedentary behavior, physical activity, and cardiorespiratory fitness on the atherogenic index of plasma. Journal of Clinical Lipidology. 2017; 11(1):119-25. [DOI:10.1016/j.jacl.2016.10.014] [PMID]

[21] Shen S, Lu Y, Dang Y, Qi H, Shen Z, Wu L, et al. Effect of aerobic exercise on the atherogenic index of plasma in middle-aged Chinese men with various body weights. International Journal of Cardiology. 2017; 230:1-5. [DOI:10.1016/j.ijcard.2016.12.132] [PMID]

[22] Naghi M, Almadadi M. [Effect of regular physical activity as a basic component of lifestyle modification on reducing major cardiovascular risk factors (Persian)]. Journal of Knowledge \& Health. 2001; 6(1):27-35. [DOI:10.22100/jkh.v6i1.92]

[23] Ghanbari-Niaki A, Saghebjoo M, Hedayati M. A single session of circuit-resistance exercise effects on human peripheral blood lymphocyte ABCA1 expression and plasma HDL-C level. Regulatory Peptides. 2011; 166(1-3):42-7. [DOI:10.1016/j.reg pep.2010.08.001] [PMID] 
[24] Sugiura H, Sugiura H, Kajima K, Mirbod SM, Iwata H, Matsuoka T. Effects of long-term moderate exercise and increase in number of daily steps on serum lipids in women: Randomised controlled trial [ISRCTN21921919]. BMC Women's Health. 2002; 2(1):3. [DOI:10.1186/1472-6874-2-3] [PMID] [PMCID]

[25] Mohammadi S, Ahmadi S, Yektayar M, Ahmadi Dehrashid K. Effects of three different modes of exercise training on plasma lipoprotein profile in healthy men. Journal of $\mathrm{Ad}-$ vances in Medicine and Medical Research. 2015; 6(5):493-9. [DOI:10.9734/BJMMR/2015/14898]

[26] Ghamarchehreh ME, Shamsoddini A, Alavian SM. Investigating the impact of eight weeks of aerobic and resistance training on blood lipid profile in elderly with non-alcoholic fatty liver disease: A randomized clinical trial. Gastroenterology and Hepatology from Bed to Bench. 2019; 12(3):190-6. [DOI:10.22037/ghfbb.v12i3.1580]

[27] Durstine JL, Grandjean PW, Cox CA, Thompson PD. Lipids, lipoproteins, and exercise. Journal of Cardiopulmonary Rehabilitation and Prevention. 2002; 22(6):385-98. [DOI:10.1097/00008483-200211000-00002] [PMID]

[28] Olson TP, Dengel D, Leon A, Schmitz K. Changes in inflammatory biomarkers following one-year of moderate resistance training in overweight women. International Journal of Obesity. 2007; 31(6):996-1003. [DOI:10.1038/sj.ijo.0803534] [PMID]

[29] Ghorbanian B, Ghasemnian A. [The effects of interval combined endurance training on some key reverse cholesterol transport factors in boy adolescents (Persian)]. Studies in Medical Sciences. 2015; 26(3):227-36. http://umj.umsu.ac.ir/ article-1-2807-en.html

[30] Ghafari G, Bolboli L, Rajabi A, Saedmochshi S. [The effect of 8 weeks aerobic training on predictive inflammatory markers of atherosclerosis and lipid profile in obese elderly women (Persian)]. Scientific Journal of Ilam University of Medical Sciences. 2016; 23(7):144-54. http://sjimu.medilam.ac.ir/article1-2147-fa.html

[31] Firozeh Z, Bizheh N, Ebrahimi Atri A, Ramezani S. [Evaluation of the efficacy of walking on estrogen hormone and some cardiovascular risk factors in non-athlete menopausal women (Persian)]. Daneshvar Medicine. 2010; 18(5):33-40. http:// daneshvarmed.shahed.ac.ir/article_1438.html?lang=en

[32] Ramos JS, Dalleck LC, Tjonna AE, Beetham KS, Coombes JS. The impact of high-intensity interval training versus moderate-intensity continuous training on vascular function: $A$ systematic review and meta-analysis. Sports Medicine. 2015; 45(5):679-92. [DOI:10.1007/s40279-015-0321-z] [PMID]

[33] Kelley GA, Kelley KS. Effects of aerobic exercise on NonHDL-C in children and adolescents: A meta-analysis of randomized controlled trials. Progress in Cardiovascular Nursing. 2008; 23(3):128-32. [DOI:10.1111/j.1751-7117.2008.00002.x] [PMID] [PMCID]

[34] Yektayar M, Mohammadi S, Ahmadi Deharshid K, Khodamoradpour $\mathrm{M}$. [Comparison of the effects of resistance, endurance and combined exercises on lipid profile of non-athlete healthy middle aged men (Persian)]. Scientific Journal of Kurdistan University of Medical Sciences. 2012; 16(4):26-36. http:// sjku.muk.ac.ir/article-1-649-en.html 
This Page Intentionally Left Blank 